\title{
An optimal instrumental variable method for continuous-time fractional model identification
}

\author{
Rachid Malti, ${ }^{*}$ Stéphane Victor, ${ }^{*}$ Alain Oustaloup, ${ }^{*}$ \\ and Hugues Garnier** \\ * IMS, UMR 5218 CNRS, Université Bordeaux1, 351 cours de la \\ Libération, F 33405 Talence cedex, France - \{rachid.malti, \\ stephane.victor, alain.oustaloup\}@laps.ims-bordeaux.fr \\ ** CRAN, Nancy-Université, CNRS, BP 239, 54506 \\ Vandoeuvre-lès-Nancy Cedex, France- \\ hugues.garnier@cran.uhp-nancy.fr
}

\begin{abstract}
: this paper deals with continuous-time system identification using fractional differentiation models in a noisy output context. The simplified refined instrumental variable for continuous-time systems (srivc) is extended to fractional models. Monte Carlo simulation analysis are used to demonstrate the performance of the proposed optimal instrumental variable scheme.
\end{abstract}

Keywords: continuous-time models; fractional differentiation; instrumental variable; state variable filter; system identification; time-domain.

\section{INTRODUCTION}

Instrumental Variable (IV) approaches to system identification and parameter estimation have been developed since 1960s and 1970s as detailed in the survey paper by Young (1981) and all references therein. Recently, Refined Instrumental Variables for Continuous-system (Young and Jakeman (1980)) has been developed for identifying hybrid continuous-time Box-Jenkins models, where the system model is continuous-time and the noise model is discrete-time (Young et al. (2006)). rivc algorithms are implemented in both Captain (Young (2006)) and Contsid (Garnier et al. (2008)) toolboxes.

The last two decades have witnessed considerable development in the use of fractional operators in various fields. Fractional differentiation is now an important tool for the international scientific and industrial communities especially in modeling viscoelastic materials and some diffusive phenomena (thermal diffusion, electrochemical diffusion). Time-domain system identification using fractional models was initiated in the late nineties and in the beginning of this century in the Ph.D. theses of Le Lay (1998); Lin (2001); Cois (2002); Aoun (2005). For an overview of different identification methods based on fractional models, refer to Malti et al. (2006).

The objective of this paper is to extend the Simplified Refined Instrumental Variable for Continuous-time systems (Young and Jakeman (1980)), denoted by srivc from hereon, to the identification of fractional models. This optimal IV method presents the advantage of yielding asymptotically efficient estimates in the presence of white measurement noise. The algorithm is initialized by a transfer function obtained from the least squares state variable filter estimates. Cois et al. (2001) are the first to extend the use of state variable filters to fractional systems. The authors suggest to use Instrumental Variables (IV) to eliminate bias. However, their instruments are not selected in an optimal way. An improvement of their method is proposed in this paper by choosing rivc algorithm. This approach involves a method of iterative-adaptive prefiltering based on a quasi-optimal ${ }^{1}$ statistical solution. The proposed method is independent from the way fractional differentiation and integration are simulated in the timedomain.

\subsection{Mathematical background}

A fractional mathematical model is based on a fractional differential equation:

$$
\begin{aligned}
& y(t)+a_{1} \mathbf{D}^{\alpha_{1}} y(t)+\cdots+a_{m_{A}} \mathbf{D}^{\alpha_{m_{A}}} y(t)= \\
& b_{0} \mathbf{D}^{\beta_{0}} u(t)+b_{1} \mathbf{D}^{\beta_{1}} u(t)+\cdots+b_{m_{B}} \mathbf{D}^{\beta_{m_{B}}} u(t),
\end{aligned}
$$

where $\left(a_{j}, b_{i}\right) \in \mathbb{R}^{2}$, differentiation orders $\alpha_{1}<\alpha_{2}<$ $\ldots<\alpha_{m_{A}}, \beta_{0}<\beta_{1}<\ldots<\beta_{m_{B}}$ are allowed to be noninteger positive numbers. The concept of differentiation to an arbitrary order (non-integer),

$$
\mathbf{D}^{\gamma} \triangleq\left(\frac{d}{d t}\right)^{\gamma} \quad \forall \gamma \in \mathbb{R}_{+}^{*},
$$

was defined in the $19^{\text {th }}$ century by Riemann and Liouville. The $\gamma$-order fractional derivative of $x(t)$ is defined as being an integer derivative of order $\lfloor\gamma\rfloor+1(\lfloor\cdot\rfloor$ stands for the floor operator) of a non-integer integral of order $\gamma-\lfloor\gamma\rfloor$ Samko et al. (1993):

\footnotetext{
1 As stated in Young et al. (2006), in the rational case, the method is quasi-optimal because true optimality would require optimal interpolation of the input signal $u(t)$ over the sampling interval, whereas only simple interpolation is used here.
} 


$$
\begin{aligned}
& \mathbf{D}^{\gamma} x(t)=\mathbf{D}^{\lfloor\gamma\rfloor+1}\left(\mathbf{I}^{\lfloor\gamma\rfloor+1-\gamma} x(t)\right) \triangleq \\
& \left(\frac{d}{d t}\right)^{\lfloor\gamma\rfloor+1}\left(\frac{1}{\Gamma(\lfloor\gamma\rfloor+1-\gamma)} \int_{0}^{t} \frac{x(\tau) d \tau}{(t-\tau)^{\gamma-\lfloor\gamma\rfloor}}\right),
\end{aligned}
$$

where $t>0, \forall \gamma \in \mathbb{R}_{+}^{*}$, and the Euler's $\Gamma$ function is defined as:

$$
\Gamma(x)=\int_{0}^{\infty} e^{-t} t^{x-1} d t \quad \forall x \in \mathbb{R}^{*} \backslash\left\{\mathbb{N}^{-}\right\} .
$$

The Laplace transform is a more concise algebraic tool generally used to represent fractional systems, see Oldham and Spanier (1974):

$$
\mathscr{L}\left\{\mathbf{D}^{\gamma} x(t)\right\}=s^{\gamma} X(s) \quad \text { if } x(t)=0 \forall t \leq 0 .
$$

This property allows to write the fractional differential equation (1), provided $u(t)$ and $y(t)$ equal 0 for all $t<0$, in a transfer function form:

$$
F(s)=\frac{B(s)}{A(s)}=\frac{\sum_{i=0}^{m_{B}} b_{i} s^{\beta_{i}}}{1+\sum_{j=1}^{m_{A}} a_{j} s^{\alpha_{j}}} .
$$

Moreover if $F(s)$ is commensurable of order $\gamma$, i.e. all differentiation orders are exactly divisible by the same number, an integral number of times (the biggest number is always chosen), then $F(s)$ can be rewritten as:

$$
F(s)=\frac{\sum_{i=0}^{n} \tilde{b_{i}} s^{i \gamma}}{1+\sum_{j=1}^{m} \tilde{a_{j}} s^{j \gamma}},
$$

where $n=\frac{\beta_{m_{B}}}{\gamma}$ and $m=\frac{\alpha_{m_{A}}}{\gamma}$ are integers and:

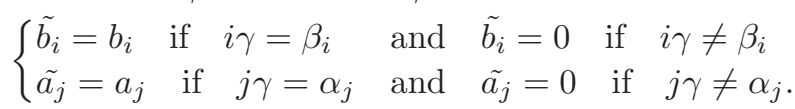

In rational transfer functions $\gamma$ equals 1 and usually numerator $\alpha_{m_{A}}$ and denominator $\beta_{m_{B}}$ orders are both fixed, then all coefficients $b_{i}, i=1, \ldots, \beta_{m_{B}}$ and $a_{j}, j=$ $1, \ldots, \alpha_{m_{A}}$ are estimated. Generally, no care is taken to check whether any intermediate coefficient, as in (7) equals zero.

Exactly the same principle will be applied here for fractional models: the commensurable order $\gamma$ together with the $\alpha_{m_{A}}$ and $\beta_{m_{B}}$ are fixed, and then all the $\tilde{b}_{i}$ and $\tilde{a}_{j}$ coefficients are estimated.

\subsection{Time-domain simulation of fractional models}

The main objective of this paper is to develop a method for time-domain system identification able to work with any fractional-model-time-domain simulation algorithm. Hence, only the simulation algorithm used in the example of this paper is explained here, as the objective is not to discuss different simulation algorithms. Refer to Aoun et al. (2004), for an extended discussion on this topic.

Due to the consideration that real physical systems generally have bandlimited fractional behavior and due to the practical limitations of input and output signals (Shannon's cut-off frequency for the upper band and the spectrum of the input signal for the lower band), fractional
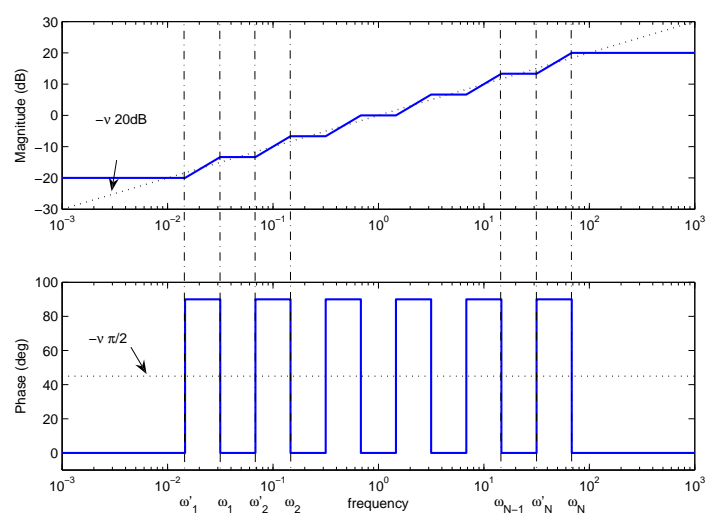

Fig. 1. Recursive approximation of a fractional differentiator with poles and zeroes

operators are usually approximated by high order rational models. As a result, a fractional model and its rational approximation have the same dynamics within a limited frequency band. The most commonly used approximation of $s^{\nu}$ in the frequency band $\left[\omega_{A}, \omega_{B}\right]$ is the recursive distribution of zeros and poles proposed by Oustaloup (1995) (see Fig. 1):

$$
s^{\gamma} \rightarrow s_{\left[\omega_{A}, \omega_{B}\right]}^{\gamma}=C_{0}\left(\frac{1+\frac{s}{\omega_{A}}}{1+\frac{s}{\omega_{B}}}\right)^{\gamma} \approx C_{0} \prod_{k=1}^{N} \frac{1+\frac{s}{\omega_{k}^{\prime}}}{1+\frac{s}{\omega_{k}}}
$$

where $\omega_{i}=\alpha \omega_{i}^{\prime}, \quad \omega_{i+1}^{\prime}=\eta \omega_{i}^{\prime} \quad$ and

$$
\gamma=1-\frac{\log \alpha}{\log \alpha \eta}
$$

$\alpha$ and $\eta$ define the differentiation order $\gamma$. The bigger $N$ the better the approximation of the differentiator $s^{\nu}$.

\section{SYSTEM IDENTIFICATION}

The Refined Instrumental Variables for Continuous system (Young and Jakeman (1980)) (rivc) method and its simplified version srivc (when the additive measurement noise is assumed to be white) are the only IV methods that can be interpreted in optimal statistical terms, so providing an estimate of the parametric error covariance matrix and, therefore, estimates of the confidence bounds on the parameter estimates. Optimal instrumental variables are obtained from an auxiliary model which is iteratively updated. At the first iteration, the auxiliary model is initialized using a least squares estimate applied on state variable filters (SVF). The identification problem is first stated, then SVF method is briefly recalled and finally the srivc algorithm is extended to fractional models.

\subsection{Problem formulation}

Consider input $u(t)$ and output $y^{*}(t)$ data collected at regular samples $T_{s}, 2 T_{s}, \ldots, K T_{s}$ from $t=0$ to $t=T$. The measured output is supposed to be corrupted by an additive measurement noise $p(t)$ :

$$
y^{*}(t)=y(t)+p(t)
$$

where $y(t)$ is the hypothetical noise-free deterministic system output. The input $u(t)$ and the output $y(t)$ signals are supposed to be related by the fractional differential equation (1). 
Equation error can be formulated as:

$$
\varepsilon(t)=y^{*}(t)-\varphi^{*}(t)^{T} \theta
$$

where

$$
\varphi^{*}(t)^{T}=\left[\begin{array}{c}
\mathbf{D}^{\beta_{0}} u(t) \cdots \mathbf{D}^{\beta_{m_{B}}} u(t) \\
-\mathbf{D}^{\alpha_{1}} y^{*}(t) \cdots-\mathbf{D}^{\alpha_{m_{A}}} y^{*}(t)
\end{array}\right]
$$

and

$$
\theta^{T}=\left[\begin{array}{lllllll}
b_{0} & b_{1} & \ldots & b_{m_{B}} & a_{1} & \ldots & a_{m_{A}}
\end{array}\right] .
$$

Minimizing the $L_{2}$ norm of $\varepsilon(t)$ :

$$
J=\int_{0}^{T}(\varepsilon(t))^{2} d t
$$

with respect to $\theta$, leads to the following least squares estimates:

$$
\hat{\theta}_{L S}=\left[\int_{0}^{T} \varphi^{*}(t)^{T} \varphi^{*}(t) d t\right]_{0}^{-1} \int_{0}^{T} \varphi^{*}(t)^{T} y^{*}(t) d t .
$$

Or, after a numerical discretization, by defining $\mathbf{Y}$ as a column vector of the system output and $\boldsymbol{\Phi}$ as a regression matrix where the columns are fractional derivatives of the input and the output signals:

$$
\boldsymbol{\Phi}^{*}=\left[\begin{array}{lll}
\varphi^{*}\left(T_{s}\right) & \varphi^{*}\left(2 T_{s}\right) & \ldots \varphi^{*}\left(K T_{s}\right)
\end{array}\right]^{\mathrm{T}},
$$

$\hat{\theta}_{\mathbf{L S}}$ can be approximated by:

$$
\hat{\theta}_{L S}=\left(\boldsymbol{\Phi}^{* \mathrm{~T}} \boldsymbol{\Phi}^{*}\right)^{-1} \boldsymbol{\Phi}^{* \mathrm{~T}} \mathbf{Y}^{*}
$$

Direct fractional differentiations of noisy output lead however to inaccurate results. Consequently, state variable filters are preferred to the direct least squares estimates.

\subsection{Least Squares-based SVF method (lssvf)}

As in the integer case, fractional differentiation of noisy signals amplifies the noise. Hence, a linear transformation (low-pass filter) is applied to input and output signals (Cois et al. (2001)). Poisson's filters can, for example, be chosen such as:

$$
F_{\gamma}(s)=\frac{s^{\gamma}}{\left(\left(\frac{s}{\omega_{c}}\right)^{\gamma}+1\right)^{N}} .
$$

The order $N$ is usually chosen such that $\gamma N>\alpha_{m_{A}}$. The basic idea in using Poisson's filter is to obtain the behavior of a differentiator in low frequencies and to filter noise in high frequencies as shown in the Bode diagram of Fig. 2. However, this filter introduces a phase shift near the cut-off frequency. The approximation is usually good enough to initialize the srivcf algorithm. Hence, differentiated filtered input $\mathbf{D}^{\beta_{i}} u_{f}$ and output $\mathbf{D}^{\alpha_{j}} y_{f}$ signals are obtained at the output of the filters (18):

$$
\left\{\begin{array}{l}
\mathbf{D}^{\beta_{i}} u_{f}(t)=u(t) * \mathscr{L}^{-1}\left\{F_{\beta_{i}}(s)\right\} \\
\mathbf{D}^{\alpha_{j}} y_{f}^{*}(t)=y^{*}(t) * \mathscr{L}^{-1}\left\{F_{\alpha_{j}}(s)\right\},
\end{array}\right.
$$

where $*$ and $\mathscr{L}^{-1}$ stand respectively for the convolution operator and the inverse Laplace transform.

Instead of minimizing the $L_{2}$ norm of $\varepsilon(t)$ as in (11), the $L_{2}$ norm of $\varepsilon_{f}(t)$ is now minimized:

$$
\varepsilon_{f}(t)=y_{f}^{*}(t)-\varphi_{f}^{*}(t)^{T} \theta,
$$

where

$$
\varphi_{f}^{*}(t)^{T}=\left[\begin{array}{c}
\mathbf{D}^{\beta_{0}} u_{f}(t) \cdots \mathbf{D}^{\beta_{m_{B}}} u_{f}(t) \\
-\mathbf{D}^{\alpha_{1}} y_{f}^{*}(t) \cdots-\mathbf{D}^{\alpha_{m}} y_{f}^{*}(t)
\end{array}\right] .
$$
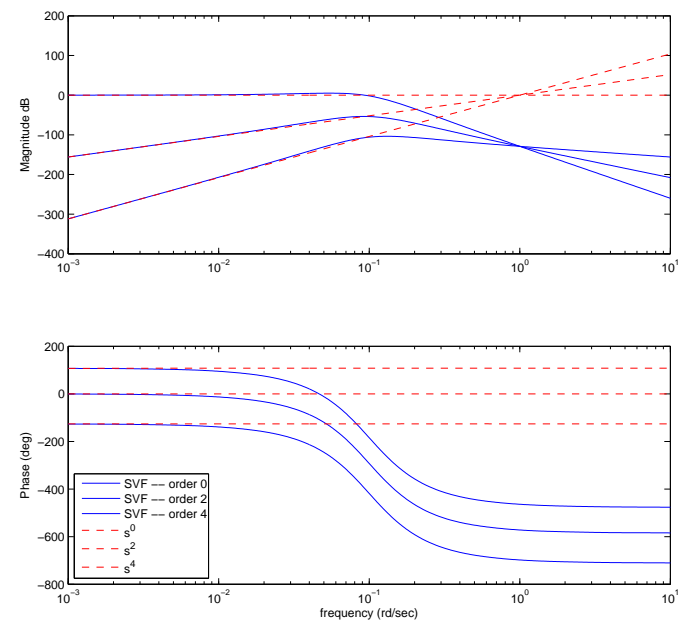

Fig. 2. Bode diagram of the fractional state variable filters

The solution is approximated (due to the integral) by the classical least squares-based SVF as in (17):

$$
\hat{\theta}_{\mathrm{LSSV}}=\left(\boldsymbol{\Phi}_{f}^{* \mathrm{~T}} \boldsymbol{\Phi}_{f}^{*}\right)^{-1} \boldsymbol{\Phi}_{f}^{* \mathrm{~T}} \mathbf{Y}_{f}^{*}
$$

where

$$
\boldsymbol{\Phi}_{f}^{*}=\left[\begin{array}{lll}
\varphi_{f}^{*}\left(T_{s}\right) & \varphi_{f}^{*}\left(2 T_{s}\right) & \ldots \varphi_{f}^{*}\left(K T_{s}\right)
\end{array}\right]^{T} .
$$

As in the integer case, Cois et al. (2001) showed that the least squares estimator $(22)$ is biased in presence of noisy output due to the correlation between $\varphi_{f}^{*}(t)$ and $y^{*}(t)$.

\subsection{Instrumental Variable-based SVF method (ivsvf)}

The estimator of the instrumental variable is a traditional variant of the least squares method. It presents the advantage of relying on linear regression techniques. The principle of the instrumental variable method relies on the so called instruments generally obtained from a parallel model $y_{f}^{I V}$. Hence, an instrumental variable regression vector is built:

$$
\varphi_{f}^{I V}(t)^{T}=\left[\begin{array}{c}
\mathbf{D}^{\beta_{0}} u_{f}(t) \cdots \mathbf{D}^{\beta_{m_{B}}} u_{f}(t) \\
-\mathbf{D}^{\alpha_{1}} y_{f}^{I V}(t) \cdots-\mathbf{D}^{\alpha_{m_{A}}} y_{f}^{I V}(t)
\end{array}\right] .
$$

The conditions that the instrumental variable vector should satisfy, are the following (see e.g. Ljung (1999)) (E[.] stands for the mathematical expectation):

$$
\left\{\begin{array}{l}
\mathrm{E}\left[\varphi_{f}^{I V}(t) \varphi_{f}^{* T}(t)\right] \quad \text { is non singular } \\
\mathrm{E}\left[\varphi_{f}^{I V}(t) p(t)\right]=0 .
\end{array}\right.
$$

Hence, the instruments, gathered in $\varphi_{f}^{I V}$, need to be sufficiently correlated to all components of the regression vector $\varphi_{f}^{*}$, and totally uncorrelated to the output noise $p(t)$.

The IV-based solution is now approximated by:

$$
\hat{\theta}_{\mathrm{IVSVF}}=\left(\boldsymbol{\Phi}_{f}^{I V} \boldsymbol{\Phi}_{f}^{*}\right)^{-1} \boldsymbol{\Phi}_{f}^{I V^{\mathrm{T}}} \mathbf{Y}_{f}^{*}
$$

where

$$
\boldsymbol{\Phi}_{f}^{I V}=\left[\begin{array}{llll}
\varphi_{f}^{I V}\left(T_{s}\right) & \varphi_{f}^{I V}\left(2 T_{s}\right) & \ldots & \varphi_{f}^{I V}\left(K T_{s}\right)
\end{array}\right]^{T} .
$$




\subsection{Simplified refined instrumental variable for} continuous-time fractional models (srivcf)

As shown by Young (1981), the optimal estimator (asymptotically without bias and of minimal variance) is obtained when the SVF filters $F_{\gamma}(s)$ in (18) are replaced by:

$$
F_{\gamma}^{o p t}(s)=\frac{s^{\gamma}}{H(s) A(s)},
$$

where $H(s)$ is the real noise model, $A(s)$ the denominator of the transfer function of the real system; and the hypothetical noise free deterministic output $y(t)$ is used when evaluating the derivatives of the output in (19). As a result, the optimal regression vector is obtained:

$$
\varphi_{f}^{\mathrm{opt}}(t)^{T}=\left[\begin{array}{c}
\mathbf{D}^{\beta_{0}} u_{f}(t) \cdots \mathbf{D}^{\beta_{m_{B}}} u_{f}(t) \\
-\mathbf{D}^{\alpha_{1}} y_{f}(t) \cdots-\mathbf{D}^{\alpha_{m_{A}}} y_{f}(t)
\end{array}\right] .
$$

The optimal estimator necessitates to know many characteristics of the system to be identified. It will be obtained only if the real system and noise models are known; however the optimality is not achievable in practical cases.

A stochastic approach proposed by Young (1981) and Young (2002) is the Simplified Refined Instrumental Variable for Continuous systems (srivc). This approach includes an adaptive pre-filtering based on the optimal solution when the noise $p$ is a white gaussian one $(H(s)=1)$. This method is a logical extension of the instrumental variable estimator with SVF. This estimator is extended to fractional models and is named srivcf (srivc for fractional models).

To optimize the instruments, it is required to implement an iterative algorithm. This algorithm can be initialized by the $\hat{\theta}_{\mathrm{LSSVF}}$ or $\hat{\theta}_{I V S V F}$ estimates (22). Once the first estimation of the denominator is available, the new derivatives of input and output signals are computed by replacing $F_{\gamma}(s)$ in $(18)$ by:

$$
F_{\gamma}^{(\text {iter })}(s, \hat{\theta})=\frac{s^{\gamma}}{\hat{A}(s)}=\frac{s^{\gamma}}{1+\sum_{j=1}^{m_{A}} \hat{a}_{j} s^{\alpha_{j}}},
$$

with $\hat{a}_{j}$ the estimated $a_{j}$ coefficient and (iter) $=1,2, \ldots$ stands for the iteration number.

The instruments, the derivatives of the input and of the output, are computed at each iteration by:

$$
\left\{\begin{array}{l}
\mathbf{D}^{\beta_{i}} u_{f}(t)=u(t) * \mathscr{L}^{-1}\left\{F_{\beta_{i}}^{(\text {iter })}(s)\right\} \\
\mathbf{D}^{\alpha_{j}} y_{f}^{*}(t)=y^{*}(t) * \mathscr{L}^{-1}\left\{F_{\alpha_{j}}^{(\text {iter })}(s)\right\} \\
\mathbf{D}^{\alpha_{j}} y_{f}^{I V}(t)=y^{I V}(t) * \mathscr{L}^{-1}\left\{F_{\alpha_{j}}^{(\text {iter }}(s)\right\} .
\end{array}\right.
$$

The regression vectors $\varphi_{f}^{*}(t)$ and $\varphi_{f}^{I V}(t)$ are formed as in $(21)$ and $(24)$. Finally, the parameter vector $\hat{\theta}_{\text {srivcf }}^{(\text {iter })}$ is computed at each iteration as:

$$
\hat{\theta}_{\text {srivcf }}^{(\text {iter })}=\left(\boldsymbol{\Phi}_{f}^{I V^{\mathrm{T}}} \boldsymbol{\Phi}_{f}^{*}\right)^{-1} \boldsymbol{\Phi}_{f}^{I V^{\mathrm{T}}} \mathbf{Y}_{f}^{*} .
$$

As in the rational case, the srivcf estimator is asymptotically unbiased whatever additive zero-mean noise is present. When the additive noise is white, srivcf has minimum variance and the asymptotic covariance matrix of the estimation errors associated with the estimate of $\hat{\theta}_{\text {srvicf }}$ is given by:

$$
\mathbf{P}_{\hat{\theta}}=\hat{\sigma}^{2}\left(\Phi^{I V^{T}} \Phi^{I V}\right)^{-1}
$$

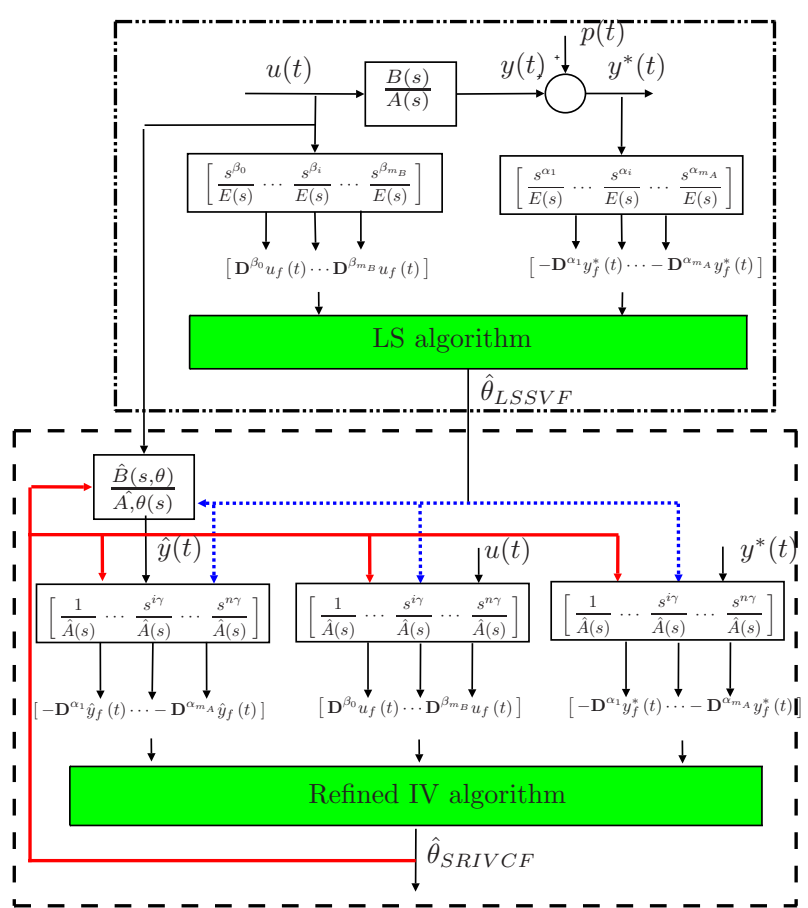

Fig. 3. Iterative optimal srivcf

where $\hat{\sigma}^{2}$ is the empirical estimation of the noise variance.

\subsection{Summary of the srivcf algorithm}

The overall srivcf algorithm is summarized in this section and sketched in Fig. 3.

Step 1 use the fractional state variable filter estimates $(22)$ to generate an initial (iter $=1$ ) estimate of the transfer function model parameter vector $\theta^{(1)}$.

Step 2 iterative instrumental variable estimation with prefilters.

for iter $=2$ to convergence

(i) Generate the instrumental variables $y^{I V}$ from the auxiliary model with the estimated polynomials based on the estimated parameter vector $\theta^{\text {(iter-1) }}$.

(ii) Update the filter $F_{\gamma}^{(\text {iter })}(s, \hat{\theta})$ in $(30)$ with the new estimated parameters. Then, evaluate the prefiltered derivatives of $u(t), y(t)$ and $y^{I V}(t)$ as in (31).

(iii) Based on these prefiltered data, compute the new estimates $\theta^{\text {(iter) }}$ as in (32).

\section{end for}

Step 3 compute the estimated parametric error covariance matrix associated with the parameter estimates with (33).

It may happen, when the bias is important, that the estimated parameters from Step 1 lead to unstable models. In this case, an empirical method can be used to stabilize unstable poles.

\section{NUMERICAL EXAMPLE}

\subsection{System specifications}

To illustrate the efficiency of this algorithm in system identification using rational and/or fractional models, the following system is simulated: 

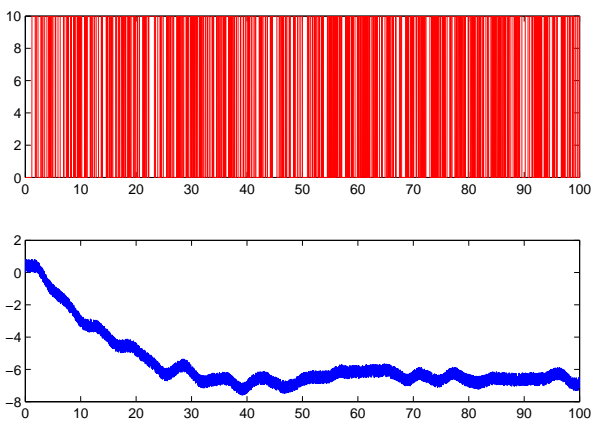

Fig. 4. Input and noisy output signals used for identification.

$$
G_{0}(s)=\frac{K\left(-T s^{\nu}+1\right)}{\left(\left(\frac{s}{\omega_{1}}\right)^{2 \nu}+2 \zeta_{1}\left(\frac{s}{\omega_{1}}\right)^{\nu}+1\right)\left(\left(\left(\frac{s}{\omega_{2}}\right)^{2 \nu}+2 \zeta_{2}\left(\frac{s}{\omega_{2}}\right)^{\nu}+1\right)\right)},
$$

with $\nu=0.5, K=-1, T=0.5, \omega_{1}=0.2 \mathrm{rad} / \mathrm{s}$, $\zeta_{1}=-0.4, \omega_{2}=1 \mathrm{rad} / \mathrm{s}$ and $\zeta_{2}=-0.65$.

This fractional system has two resonant modes: one at $\omega_{1}=0.2 \mathrm{rad} / \mathrm{s}$ with $\zeta_{1}=-0.4$ and another one at $\omega_{2}=1$ $\mathrm{rad} / \mathrm{s}$ with $\zeta_{2}=-0.65$ (for stable fractional systems, a resonant peak may occur for negative pseudo-damping factors $\left.\zeta_{1,2}^{2}\right)$. Furthermore, the system has a positive $s^{\nu}$ zero at $s^{\nu}=2$.

The input signal $u(t)$, a pseudo random binary sequence (prbs), is applied to the system (34). The prbs amplitude is intentionally chosen between 0 to 10 in order to be able to capture the rapid dynamics and the long memory behaviour (slow dynamics) typically present in fractional differentiation systems. The output, $y(t)$, is corrupted by an additive Gaussian white noise $p(t)$ with zero mean and a signal to noise ratio $(\mathrm{S} / \mathrm{N})$ of $20 \mathrm{~dB}$ as shown in Fig. 4. The sampling period is fixed to $T_{s}=10^{-2} \mathrm{~s}$.

The instrumental variables are computed according to the iterative algorithm described in $\S 2.5$ in order to rippen the parametric estimation. The model, which parameters are to be estimated, is set to:

$$
G(s)=\frac{b_{1} s^{\nu}+b_{0}}{a_{4} s^{4 \nu}+a_{3} s^{3 \nu}+a_{1} s^{2 \nu}+a_{1} s^{\nu}+1} .
$$

To be in the same class as the true system, the commensurable order must be set to 0.5. However, the commensurable order is not always known; when unknown, the optimal commensurable order can be estimated.

\subsection{The choice of commensurable order}

At the first stage, srivcf is applied with commensurable orders varying from $\nu=0.1$ to $\nu=1.9$ with a step of 0.05 . The log-normalized cost function

$$
J_{\mathrm{dB}}=10 \log _{10}\left(\frac{\sum_{k=1}^{K}\left(y_{m}\left(k T_{s}\right)-y^{*}\left(k T_{s}\right)\right)^{2}}{\sum_{k=1}^{K} y_{m}\left(k T_{s}\right)^{2}}\right),
$$

where $y_{m}$ stands for the model output, is plotted versus the commensurable order in the region of interest, i.e. $\nu \in[0.3,1]$, in Fig. 5 . As expected, the best commensurable

\footnotetext{
2 When $\nu \neq 1, \zeta$ cannot be considered as a damping factor
}

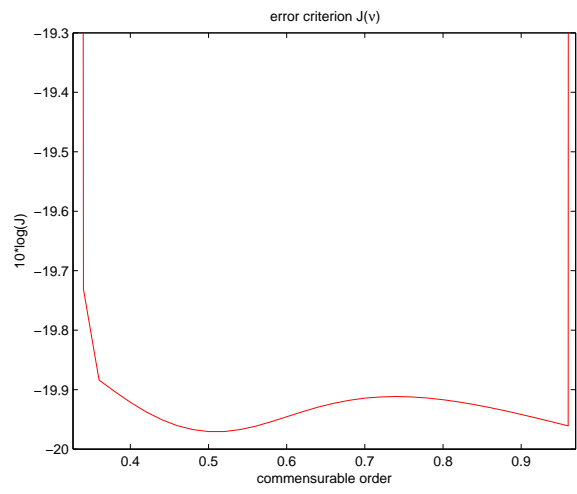

Fig. 5. Cost function in $\mathrm{dB}$ vs the commensurable order.

\begin{tabular}{|l|l|l|l|l|l|}
\cline { 2 - 6 } \multicolumn{1}{c|}{} & true & \multicolumn{2}{|c|}{$\begin{array}{c}\text { srivcf } \\
(\nu=0.5)\end{array}$} & \multicolumn{2}{l|}{ ivsvf } \\
\cline { 2 - 6 } \multicolumn{1}{c|}{} & & $E(\hat{\theta})$ & $\sigma(\hat{\theta})$ & $E(\hat{\theta})$ & $\sigma(\hat{\theta})$ \\
\hline$a_{4}$ & 25 & 25.037 & 0.026 & 25.149 & 0.165 \\
\hline$a_{3}$ & -36.5 & -36.546 & 0.029 & -36.671 & 0.106 \\
\hline$a_{2}$ & 31.2 & 31.235 & 0.015 & 31.252 & 0.034 \\
\hline$a_{1}$ & -5.3 & -5.302 & 0.004 & -5.311 & 0.006 \\
\hline$b_{1}$ & 0.5 & 0.500 & 0.002 & 0.502 & 0.015 \\
\hline$b_{0}$ & -1 & -1.002 & 0.001 & -0.999 & 0.002 \\
\hline
\end{tabular}

Table 1. Comparison between srivcf and ivsvf methods, $E(\hat{\theta})$ being the mean and $\sigma(\hat{\theta})$ the standard deviation

order is at $\nu=0.5$, accordingly to the true system (34).

\subsection{Comparison between ivsvf and srivcf methods}

This comparison will be done when the model (35) is in the same class as the true system, with $\nu=0.5$. The objective here is to study the performance of the srivcf method as compared to the ivsuf method (Cois et al. (2001)). With different noise realizations with a $\mathrm{S} / \mathrm{N}=20 \mathrm{~dB}$, a Monte Carlo simulation of 200 runs has been performed. For each realization of noise, two models were evaluated: one with ivsvf, and another with srivcf. The Monte Carlo simulation results are displayed in Table 1.

Even though ivsvf and srivcf methods are both asymptotically unbiased (Young (2002)), srivcf significantly improves the parameter variance, as displayed in the $\sigma(\hat{\theta})$ columns (standard deviation) of Table 1.

The frequency responses of the whole set of models obtained with srivcf and ivsvf methods are plotted in Fig. (6- $a$ ) and (6- $b$ ) respectively. As expected the 200 models obtained with srivcf fit exactly the Bode diagram of the simulated system (34) as compared to the ivsvf ones which have a larger dispersion.

\subsection{Comparison between a fractional model and a rational one both evaluated with srivcf method}

Considering srivcf, rational models are compared to fractional ones using a Monte Carlo simulation of 200 runs with $\mathrm{S} / \mathrm{N}=20 \mathrm{~dB}$. For each realization of noise, two models are evaluated: one for the best commensurable order $\nu=0.5$, and the other for a rational model with $\nu=1$. 

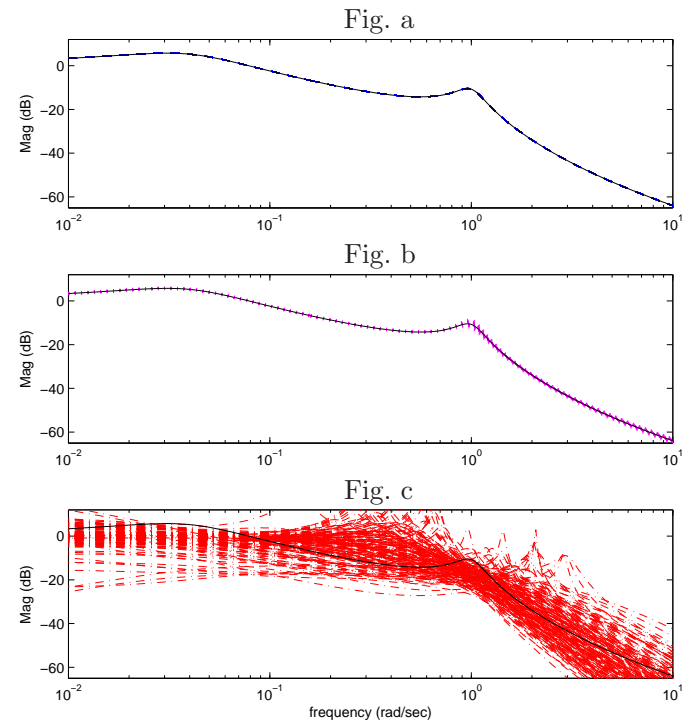

Fig. 6. Magnitude Bode diagrams of the models estimated for the 200 Monte Carlo simulation runs - Figure a: the true system $(-)$, the fractional models $(\nu=0.5)$ obtained with srivcf (-- lines) - Figure b: the true system $(-)$, the fractional models $(\nu=0.5)$ obtained with ivsvf (-- lines) - Figure c: the true system $(-)$, and the rational models $(\nu=1)$ obtained with $\operatorname{srivcf}(-.-$ lines $)$.

For $\nu=0.5$, the model coefficients $b_{i}$ and $a_{j}$ in (35) converged to the exact values (34) for all 200 Monte Carlo runs. However, for $\nu=1$, unstable and inappropriate models are obtained. The frequency responses of the whole set of 200 rational models obtained for $\nu=1$ are plotted in Fig. (6-c). As expected, the rational models give less satisfactory results as the asymptotic slops of rational systems are multiples of $20 \mathrm{~dB}$ per decade in the magnitude Bode diagram, compared to fractional models which can have any asymptotic slope (Oustaloup (1995)). If a rational model equivalent to a fractional model in a desired frequency range were to be obtained, then a much higher order model would be required.

\section{CONCLUSION AND OUTLOOKS}

In this paper, the simplified refined instrumental variable for continuous-time systems has been extended to fractional models (srivcf). The srivcf iterative algorithm is initialized from the traditional least squares-based SVF estimates. Numerical simulations have illustrated the performance of the proposed method.

The proposed approach can be seen to have a single tuning parameter: the commensurable order. As shown in the example, the cost function is tightly linked to the commensurable order. More studies are yet to be done on fractional models. Here, only the coefficients were estimated; the parametric estimation could be extended to the commensurable order. An interesting perspective would be to evaluate the procedure based on the properties of the instrumental product matrix (see Young and Jakeman (1980) or Young et al. (2006)) for identifying the commensurable model prior to parameter estimation. Another interesting perspective is to develop a rivcf algorithm for hybrid Box-Jenkins models as in rational systems (Young et al. (2006)) in order to obtain minimum variance parameters in presence of colored noise.

\section{REFERENCES}

M. Aoun. Systèmes linéaires non entiers et identification par bases orthogonales non entières. $\mathrm{PhD}$ thesis, Université Bordeaux 1, Talence, 2005.

M. Aoun, R. Malti, F. Levron, and A. Oustaloup. Numerical simulations of fractional systems: an overview of existing methods and improvements. An Int. J. of Nonlinear Dynamics and Chaos in Engineering Systems. Special issue: Fractional Derivatives and Their Applications., 38(1-4):117-131, 2004.

O. Cois. Systèmes linéaires non entiers et identification par modèle non entier : application en thermique. $\mathrm{PhD}$ thesis, Université Bordeaux 1, Talence, 2002.

O. Cois, A. Oustaloup, T. Poinot, and J.-L. Battaglia. Fractional state variable filter for system identification by fractional model. In IEEE 6th European Control Conference (ECC'2001), Porto, Portugal, 2001.

H. Garnier, M. Gilson, T. Bastogne, and M. Mensler. Identification of continuous-time models from sampled data, chapter CONTSID toolbox: a software support for continuous-time data-based modelling. Springer-Verlag, H. Garnier and L. Wang (Eds.) edition, 2008.

L. Le Lay. Identification fréquentielle et temporelle par modèle non entier. $\mathrm{PhD}$ thesis, Université Bordeaux I, Talence, 1998.

J. Lin. Modélisation et identification de systèmes d'ordre non entier. PhD thesis, Université de Poitiers, France, 2001.

L. Ljung. System identification - Theory for the user. Prentice-Hall, Upper Saddle River, N.J., USA, 2 edition, 1999.

R. Malti, M. Aoun, J. Sabatier, and A. Oustaloup. Tutorial on system identification using fractional differentiation models. In 14th IFAC Symposium on System Identification (SYSID'2006), Newcastle, Australia, 2006.

K.B. Oldham and J. Spanier. The fractionnal calculus. Academic Press, New-York and London, 1974.

A. Oustaloup. La dérivation non-entière. Hermès - Paris, 1995.

S.G. Samko, A.A. Kilbas, and O.I. Marichev. Fractional integrals and derivatives: theory and applications. Gordon and Breach Science, 1993.

P.C. Young. Optimal IV identification and estimation of continuous-time TF models. In 15th World IFAC Congress, Barcelona (Spain), 2002. IFAC, Elsevier.

P.C. Young. The captain toolbox for matlab. 14th IFAC Symposium on System Identification (SYSID'2006), 2006. Newcastle, Australia.

P.C. Young. Parameter estimation for continuous-time models - a survey. Automatica, 17(1):23-29, 1981.

P.C. Young and A.J. Jakeman. Refined instrumental variable methods of time-series analysis: Part iii, extensions. International Journal of Control, 31:741-764, 1980.

P.C. Young, H. Garnier, and M. Gilson. An optimal instrumental variable approach for identifying hybrid continuous-time box-jenkins models. In 14 th IFAC Symposium on System Identification (SYSID'2006), pages 225-230, Newcastle, Australia, 2006. 